\title{
Transcutaneous Bilirubin for Screening of Significant Jaundice in Very Preterm Neonates
}

\author{
Deepak Chawla $^{1}$ iD
}

Received: 22 September 2018 / Accepted: 4 October 2018 / Published online: 29 October 2018

(C) Dr. K C Chaudhuri Foundation 2018

Neonatal jaundice is the most common morbidity in term infants. Preterm neonates are at even higher risk of developing jaundice requiring treatment. [1] Accurate measurement of bilirubin levels and appropriate therapeutic response are essential to prevent the rise of bilirubin to potentially neurotoxic levels. Clinical assessment of the severity of hyperbilirubinemia is done by inspection of skin for cephalocaudal progression of yellowish discoloration and intensity of "yellowness". However, due to low sensitivity of the clinical visual assessment, measurement of bilirubin levels by transcutaneous bilirubinometer is the recommended method of screening for detection of significant hyperbilirubinemia. While there are strong evidence-based recommendations about application of transcutaneous bilirubin $(\mathrm{TcB})$ for detection of jaundice in term and later preterm neonates, no similar recommendations exist for very preterm neonates. [2]

Therapeutic threshold for starting treatment is not only understandably lower in preterm neonates, but also has a broad range with a sliding scale depending on gestation at birth and presence of comorbidities. [3] Combined with an expected error of $\pm 2-3 \mathrm{mg} / \mathrm{dL}$ for agreement between concurrent $\mathrm{TcB}$ and serum total bilirubin (STB) values, it is difficult to rule out or rule in significant jaundice based on TcB alone, thus necessitating the measurement of STB. As a result, despite a number of studies proving good correlation between STB and TcB in preterm or very low birth weight neonates, use of TcB has not found widespread use in this population. [4]

Agrawal et al. in a study published in this issue of the journal, evaluate the correlation and diagnostic performance of TcB measured at different body sites with STB in preterm neonates born at less than 35 wks of gestation. [5] TcB

Deepak Chawla

drdeepakchawla@gmail.com

1 Department of Neonatology, Government Medical College Hospital, Chandigarh 160 030, India measured at interscapular area was found to have best correlation with STB and highest sensitivity for diagnosing the need of phototherapy. However, even for intrascapular area, the $95 \%$ CI for agreement between STB and TcB ranged from -4.3 to $+5.4 \mathrm{mg} / \mathrm{dL}$. These values of bias are higher than those reported from studies included in a systematic review and those reported from India. [4, 6] Due to wide margin of error in the study, the negative predictive value (NPV) for diagnosing the need of phototherapy was only $64.5 \%$. Correlation and agreement between TcB and STB may differ depending on postnatal age and level of STB. A subgroup analysis evaluating agreement between the two measurements at lower STB (e.g., $10 \mathrm{mg} / \mathrm{dL}$ ) would be useful to assess the utility of TcB as a screening tool. A screening test like TcB should have a high (>95\%) NPV to justify its use in clinical practice for very preterm neonates.

\section{References}

1. Narang A, Kumar P, Kumar R. Neonatal jaundice in very low birth weight babies. Indian J Pediatr. 2001;68:307-9.

2. American Academy of Pediatrics Subcommittee on Hyperbilirubinemia. Management of hyperbilirubinemia in the newborn infant 35 or more weeks of gestation. Pediatrics. 2004;114:297-316.

3. Maisels MJ, Watchko JF, Bhutani VK, Stevenson DK. An approach to the management of hyperbilirubinemia in the preterm infant less than 35 weeks of gestation. J Perinatol. 2012;32:660-4.

4. Nagar G, Vandermeer B, Campbell S, Kumar M. Reliability of transcutaneous bilirubin devices in preterm infants: a systematic review. Pediatrics. 2013;132:871-81.

5. Agrawal G, Garg K, Sitaraman S, Sarna A. Comparison of diagnostic accuracy of different sites for transcutaneous bilirubin measurement in early preterm infants. Indian J Pediatr. 2018. https://doi.org/10. 1007/s12098-018-2739-4.

6. Chawla D, Jain S, Kaur G, Sinhmar V, Guglani V. Accuracy of transcutaneous bilirubin measurement in preterm low-birth-weight neonates. Eur J Pediatr. 2014;173:173-9. 